but on June 12th, having been attacked with measles, he was removed upstairs to the fiever ward.

June 29th. He was readmitted. The swelling was now of about the same size as at the last report (June 12th).

July 1st. The tumour was again punctured; but, owing to the toughness of the cyst and the bluntness of the trocar, little more than the end of the cannula was introduced into the interior of the tumour, and very little iodine could be injected. The fluid which came away was slightly tinged with blood, but much thinner than last time it was tapped.

July 2nd. There was no bleedingr. 'The cyst was collapsed, and contained more solid matter.

July 17th. There was no further increase in size. The child was ordered to go home, and to be brought back again if the cyst enlarged.

August (latter end). He was seen as an out-patient. No increase in size had occurred. The tumour was quite solid, consisting only of an enlarged gland, of about the size of a walnut.

Remanks. The above are instances of a disease which frequently proves very intractable, especially in adults, and which, from the situation, size, and connexions of the tumour, may in such cases become one of the most formidable ailments which a surgeon can be called upon to treat. A very good illustration of this fact was furnished by the case of a young woman who was a patient at St. George's Hospital several years ago, with a large serous cyst (hydrocele) of the neck, and in whom the tumour resisted for $n_{1}, \rightarrow$ than a year all the attempts made to produce its obliteration. Thus it was originally tapped and in. jected, as in the two cases here reported; then, as, after repeated injections, it continued to fill again, a seton was intropuced; and, on this measure failing, it was laid open from top to bottom, and the lining membrane destroyed, as far as possible, with nitric acid. The cyst was still, however, undestroyed, and was apparently reproduced by growth from below. Accordingly, it was again cut down upon, and an attempt was made to dissect it out entire. This, however, was found to be impossible, from the membrane dipping below the sheath of the carotid vessels. Accordingly, the wound was kept permanently open, and the cyst gradually contracted, and at length, it is believed, became perfectly obliterated. In such a case as this, it is evident that the cyst must have had some origin more deeply seated than the common areolar tissue, but whether it was connected with the thyroid body, or not, seemed uncertain. The origins which are generally ascribed to such tumours, when they are not merely subcutaneous, are, that they are formed by dilatation of the cavities of the thyroid gland, or from cysts in connexion with the blood-vessels, either in vascular tumours or otherwise. (See Paget, Lectures on Surgical Pathology, vol.ii, p. 38.) In the two cases before us, it seemed most likely that the cysts had no such origin, and that the admixture of blood which the fluid contained was produced merely by exudation from the lining membrane of an otherwise simple serous cyst. It will be noticed, however, that in Mr. Johnson's case, after an apparent obliteration of the cyst, it rapidly tilled arain during one night, probably from the effusion of blood into its cavity, so that here some communication with the cavity of a blood vessel is certainly possible. In Mr. Prescott Hewett's case, the rapid and entire obliteration of the cyst by one injection argues against any deeper origin for the tumour, although here also the serum was mixed with blood in considerable quantities. The presence of cholesterine crystals in Mr. Johnson's case will be noticed. "Such contents", Mr. Paget says, " may occur, perhaps, in any cyst in the neck or elsewhere; but they appear to be comparatively frequent at or near the front of the larynx." An interesting circumstance in both cases is the absence of all symptoms of pain or inflamma tion after the injection of iodine into tumours which, considering the patient's age, were very large ones. The fluid did not seem even to produce pain, and the resulting consolidation was free from any indication of threatening suppuration.

Association of the Fellows and Licentiates of the Kirg and Quena's College of Physicians, Ireland. 'The following officers have been elected for the Session 1858 and 1859 :-President: Sir Henry Marsh, Bart., M.D., M.R.I.A. Vice-Presidents: Charles P. Croker, M.D., M.R.I.A. and Robert Law, M.D., M.R.I.A. Council: Drs. Henry Kennedy, John Hughes, Attinill, McClintock, and Churchill. Treasurer : Dr. George A. Kennedy. Secretary: Dr. William Moore. Meet ings of the Association are held in the College Hall, on the first Wednesday of every month, from October to June inclusive, at 8 o'clock P.Mr.

\section{ofriatinal Communitations.}

\section{ON SUPPURATION WITHIN THE CRANIUM AFTER INJURIES.}

By T. Holdres, M.A., Curator of the Museum of St. George's Hospital.

AT page $7 \pm 2$ of the Journal there is to be found a most interesting case reported by Mr. Dumville of Manchester, in which he trephined the skull on account of suppuration beneath the dura mater, with success, the patient having recovered rapidly. I was much impressed with the account of this case, having watched many such, and having observed that the uniform result, whether the operation was performed or not was fatal; and that the post mortem appearances were in all these cases such as to show that no treatment could have been of any avail. Indeed, so constant is this, that I doubt whether Mr. Dumville's case is not the only successful one which has been published of late years, perhaps since $\mathrm{Du}$ puytren's. (Chelius's Surgery, by South, vol. i, p. 399.) I was therefore surprised to see that Mr. Dumville has been led by his experience to the opinion that limited suppuration beneath the cranium " may not be so rare as some surgeons imagine"; and, as my own opinion is the direct reverse of this, believing as I do that an abscess properly so called (i.e., a limited and encysted collection of matter) below the skullcap is hardly ever met with, I thought it might not be uninteresting if I endeavoured to bring together the facts on which this opinion is founded. If this paper has the effect of fixing attention on $\mathrm{Mr}$. Dumville's case, I shall think my time worthily expended; since it is one in which (to judge from the description) ac curacy in diagnosis and a judicious boldness in treatment were combined in a degree which is rarely met with, and by which the patient was rescued from a disease otherwise inevitably fatal.

The question then being, What is the relative frequency of limited and diffused suppuration beneath the skullcap after injuries of the head?-I shall endeavour to answer it by a reference to the post mortem records of St. George's Hospital for the last seventeen years, viz, from 1841 to 1857 inclusive. A detailed account of the dissection of every case which was examined after death (and these form, at our Hospital, a very great majority, and more especially of head-cases) has been preserved, and to this is appended, during the last fourteen years, a report of the case from notes taken during life. I propose to divide these cases into four classes, viz. :-

I. Those which were trephined during life for intracranial suppuration.

II. Those in which matter was found after death between the skull and dura mater.

III. Those in which matter was found among the membranes of the brain, but below the dura mater.

Iv. Those in which the cerebral substance alone contained matter.

I. Of the first class, eight cases only occurred during the whole of the above mentioned period, and of these one was not examined. I append a succinct report of the seven in which a post mortem examination was made; in the other, the symptoms were similar, but, as it was not examined after death, I will not burden space with it.

CASE I (vol. v, p. 128). A man, aged 45 , who had received a scalp wound three weeks before death, of large extent, and exposing a great amount of the bone (right parietal). He had no symptoms till thirteen days after the accident; then appeared dull and heavy; the discharge from the wound diminished, and the granulations did not look so healthy. Next day, pain and shooting across the forehead occurred. He was put on a course of calomel. On the eighteenth day after the injury, he had a fit accompanied by rigor, and the right side of the body and face was paralysed. Two crowns of trephine were applied on the exposed bone, and yellow lymph was found between the bone and dura mater. This seems to have produced no relief (the notes are wanting from this time), and he died on the twenty-first day.

On post mortem examination, the bone was found of a dead yellow colour. Thick concrete lymph was found above the dura mater; the membrane itself was thickened by an effusion of lymph. The clots in the sinuses were mixed with pus. Large quantities of lymph and pus were found both in the arachnoid 
and subarachnoid cavities. There were secondary deposits in the lungs.

CaSE II (vol. v, p. 231). A man, aged 26, had received a blow from a falling body, inflicting a large scalp-wound over the biparietal suture, connected with a linear fracture on the left side, discovered after death. Seventeen days after the accident, he was attacked with rigors, which recurred frequently, and gave place on the twenty-first day to paralysis of the right arm, with occasional twitchings of the left side of the body. Next day, the paralysis of the right side was complete. On this day, he was trephined at the seat of injury; some pus was evacuated, probably from the diploe. He died next day, the symptoms persisting.

On post mortem examination, pus was found in the diploe, and in the longitudinal sinus, as well as in the arachnoid and subarachnoid cavities. The convolutions of the brain also appeared at one part of a darker colour than at others. Lymph was also found external to the dura mater. Secondary deposits existed in the liver.

CASE III (vol. vii, p. 244). A man, aged 25, who had had a scalp wound on the left side, near the vertex, three weeks before admission. On admission, the wound was healed, except a small sinus which led down to dead bone. He had had rigor the day before, followed by sweating. The right facial nerve was partly paralysed; the tongue somewhat drawn to the left side ; the right arm numb and almost powerless. He could not walk without assistance. He had sligh; headache. The pulse was 81 , quiet and regular; the tongue moist; the skin cool. He was purged vigorously, and on the fifth day the symptoms had all disappeared, except slight numbness of the arm. He then insisted on getting up, and next day all the symptoms recurred, with slight numbness of the right lower extremity, and pain in the left side of the head. The pulse, however, was still quiet, and the skin cool. On the eighth duy, there was almost total paralysis of the right side, and this became complete on the ninth day; the headache also was more severe; the countenance more dull and heavy; and the articulation thick and imperfect. He was then trephined over the exposed bone. The bone having been removed, some very fotid pus, mixed with brain-like matter, exuded. The dura mater was found separated for some distance, but healthy in all parts except one, where a sloughy opening, the size of the tip of the thumb, existed. Pus escaped here from beneath the membrane; about three ounces altorether were evacuated. He survived the operation five days. Pus escaped freely during this time, but the principal symptoms were not relieved. The opening in the dura mater became larger, and the brain began to protinde on the day before his death. Just previous to his death, a discharge of matter occurred from the mouth and nostrils in large quantity, and appeared to produce suffocation.

On post mortem examination, no matter was found between the bone and dura mate:. The brain fitted into the sloughy opening in the latter membrane, and the surface of the convolutions here was sloughy, and pierced by a hole leading into a large abscess in the brain, which had almost penetrated into the lateral ventricle.

CASE IV (vol. ix, p. 245). George D., aged 25, was admitted with a scalp wound a lit.le to the right side of the forehead, and another on the vertex, the latter exposing the bone. There were no special symptoms (except a slight attack of erysipelas) for the first ten days; then he had a severe rigor, followed by slight incoherence of manner and much prostration, with a little pain in the head. Next day there was also partial palsy of the left arm and leg. The scalp around the wound was puffy, but the pericranium was not separated. On the following day, he was much the same. An incision let out some matter from beneath the occipito-frontalis tendon. Next day (thirteenth after the wound) he was noticed to have some cough; his evacuations were passed involuntarily. The palsy was complete on the left side. The muscles of the right side of the neck were rigid, the features drawn to the right. In the posterior wound, the pericranium seemed a little more detached than it had been two days previously. His manner was oppressed, but he was not unconscious. The trephin was applied here, and a small quantity of pus found beneath the bone, with lymph and pus extending further than the piece re moved. Accordingly, a second crown was taken away. The operation did not in any way relieve the palsy. The delirium increased. He sank rapidly, and died next day.

The pericranium was easily separable around the trephine holes, and the bone there was vascular. Pus was found in the diploe of the bone, in the superior longitudinal sinus, above the dura mater, in the arachnoid and subarachnoidean spaces over the right hemisphere, and around the medulla oblongata ; and an abscess in the right bemisphere of the cerebrum. There was also secondary deposit in the lungs.

CASE V (vol. xi, p. 199a). A man, aged 37, who had had, four days before admission, a scalp wound, not exposing the bone, over the left side of the frontal bone. The parts. sloughed from tight pressure. Except symptoms of threatening delirium tremens (which subsided), he went on well for nineteen days; then pain in the head came on, with restlessness, hot skin, sharp pulse, foul tongue. No more distinct symptoms showed themselves till the twenty-third day, when symptoms of limited pressure came on; viz., partial unconsciousness (he would mimic gestures to which his attention was called), deviation of the tongue to the right side, and ptosis of the left eyelid. Next day he passed urine unconsciously, and on the following (twenty-fifth) day became quite urconscious. No general paralysis is mentioned. He was trephined on this day at the seat of injury. Much pus was found in the diploe, and a considerable quantity let out from under the bone. The symptoms, however, were not relieved; and he died next morning.

The veins leading from the wound were found to contain pus, as did those of the bone and the superior longitudinal sinus. Pus and lymph were found also between the dura mater and the bone, in the arachnoid cavity, and among the meshes of the pia mater. The orbital isvity and frontal sinus contained abscesses. There was also secondary deposit in the liver and lungs.

CASE vI (vol. xii, p. 169). A man, aged 40, had been an outpatient for a fortnight with a scalp wound which had been phagedænic, and which, on the separation of the slough, exposed a piece of the left temporal bone as large as a crownpiece. He went on well for nine days, when constant sickness came on accompanied by pain in the head and restlessness. There were no rigors, and the wound looked healthy. This state of things lasted a week, during which time he had cold chills occasion. ally. On the sixteenth day, the sickness was relieved, but he was delirious at night, and complained of severe pain in the head. On the eighteenth day, he was quite insensible, and completely paralysed on the right side. He was extremely weak. Three days after this, the coma becoming gradually more p:ofound, he was trephined at the seat of injury. Pus was found in the diploe of the bone only. He was not relieved by the operation, and died on the twenty-third day.

At the post mortem examination, pus was found between the bone and dura mater. The veins of the diploe and superior longitudinal sinus contained pus. Pus was also found in the subarachnoidean space on that side. There were secondary deposits in the lungs.

CaSE vir (vol. xv, p. 245). A man, aged 28 , had a small scalp wound, exposing the bone on the left side, some days previously to admission, and had neglected it. When brought to the hospital he was partially conscious; the wound was discharging profusely; the facial muscles on the right side were paralysed; the left pupil was dilated, and both insensible. During the next two days, the unconsciousness increased, the water and faces were passed involuntarily. On the fifth day after admission, paralysis of the side opposite to the injury came on. He had had numerous fits, both on that and the preceding day, affecting the muscles of both sides. He was trephined on this day at the seat of injury. Pus was found in the diploe, and a small quantity of pus below the bone, which was rough on its surface. The paralysis and fits continued during the next day, but on the following day these fits ceased and some improvement occurred. On the third day after the first operation, a puffy tumour was observed under the temporal muscle, and on cutting down on this the pericranium was found separated from the bone. The trephine was accordingly applied here, but no matter was found. He survived this last operation five days; the symptoms continued unrelieved except that consciousness partially returned, so that he would make an effort to answer when spoken to, and put out his tongue when ordered. Shortly before he died, pyæmic abscesses presented in the axilla and elbow.

On post mortem examination pus was found in the cerebral vein, and in the longitudinal and lateral sinuses, also between the bone and dura nater, in the arachnoid cavity, and in the meshes of the pia mater to a very great extent.

There were secondary deposits in the lungs, and in the shoulder and elbow joints.

We thus see that in every case in which pus was sought for 
by trephining it was found. In all these cases, however, there was the local injury as a guide in the operation, and it is only in the very rare instances, where there is no distinct spot injured, no scalp-wound or fracture, that much hesitation need be felt on this point. The course of the symptoms, the time at which they occur, and the precedence of rigors and other indications of suppuration, sufficiently distinguish between effusions of pus and those of blood or serum, and $\dot{a}$ fortiori $i$ do the symptoms differ widely from those of morbid growths. But if the surgeon will be seldom disappointed in his primary object-the discovery of matter-this series supplies a very intelligible reason why his ultimate end is so rarely attained in the preservation of the patient's life. That out of seven successive cases, six should present unmistakeable evidence of pyæmia in other parts of the body, and of phlebitis in the bones and sinuses of the skull, can hardly be considered an accidental circumstance, and will, I fear, go far to show that Mr. Dumville's opinion, as to the relative frequency of limited and diffused suppuration, is not borne out by pathological investigation. I would willingly believe, however, that this may not be so in other parts of the country, where pyæmia, that bane of all operative surgery in our London hospitals, is less rife; and I would appeal to the experience of our country hospitals to correct, if possible, this conclusion.

But it may be said, were these eight cases properly selected for the operation? Were no limited abscesses found after death, in cases which had not been operated on, either above the dura mater, amongst the membranes of the brain, or even in the brain itself? 'To answer these questions, I refer again to our post mortem books; and here I find seventeen other cases, besides the sever, which I have detailed above, in which a post morten examination showed the precise condition of the parts.

II. Eight of these belong to our second class, in which matter* was found between the bone and dura mater after death, the skull not having been trephined.

CASE I. In one (vol. viii, p. 211), the injury was a scalp wound not exposing the bone. The bone was infiltrated with pus; besides the pus between the bone and dura mater, the tissue of that membrane was infiltrated with pus, and there was matter in still greater quantity in the arachnoid and subarachnoid spaces. The blood in the longitudinal sinus was also thought to contain pus. This was in all probability a case of pyæmia ; but the other viscera were not examined.

Cases Ir and III. Two other cases followed scalp wounds, in which the bone had been exposed. In the first (vol. iv, $p$. $132)$, as the spine was fractured, no question of operating could have occurred. The mischief in the head followed an attack of diffuse inflammation. There was pus, both in the circumscribed and diffused form, between the bone and dura mater, and there was suppuration also in the arachnoid cavity. The texture of the brain had a greenish hue in places. The other (vol. v, p. 193) was a case of pyæmia with secondary deposit in the lungs. There was extensive suppuration here between the bone and dura mater; and it was noticed that this suppuration was not in any way circumscribed, although a puffy tumour existed on the scalp. There was also effusion of fluid in the arachnoid and subarachnoid spaces, and abscesses (probably pyæmic) in the orbit.

CASE IV (vol. iv, p. 270). The notes of this case during life are wanting. There was, however, slight separation of one of the sutures with exposed bone. A small quantity of pus and lymph was found between the bone and dura mater, and this might probably have been evacuated by the trephine; but large quantity of pus existed also in the arachnoid cavity; and there were secondary deposits in the lungs.

CASE v (vol. vi, p. 158). This was a case of simple fracture, with widle separation of the fragments. To this separation it was probably owing, that though there was a very extensive effusion of lymph and pus between the bone and dura mater along the whole line of injury, there were no very distinct symptoms of pressure, the patient dying of diffuse inflammation. 'There was pus also in the arachnoid and subarachnoid spaces.

CAses vi and vir. These are cases of compound fracture; in the first case (vol. vi, p. 1(is), with laceration of the brain and hernia cerebri; and in the second (vol. xiv, p. 168), with loosening of a portion of bone which had been removed. In both eases, therefore, there was free exit for the matter. Both

* I have included cases in wbich yellow puriform lymph was found, even if it had not yet softened completely into pus. This would obviously hav a tendency to make the discovery of limited deposits more probable. presented matter in the arachnoid cavity, and the latter also in the subarachnoid space.

CASE viII. Lastly (p. xii, 101), we have the same state of things in a fracture of the base of the skull; here there was pus between the bone and dura mater at the seat of fracture, and it extended also into the orbit, and into the tympanum on the affected side; there was also extensive effusion of lymph in the subarachnoid space, and pus in the sinuses. This latter feature renders it probable that pyæmia either existed or was impending.

These were all the cases in which pus existed above the dura mater; in none of them was the suppuration limited to a circumscribed abscess below the bone corresponding to a separation of the pericranium from its external surface, as described by Pott; nay, further, in the only two cases in which anything resembling this "puffy tumour" was seen, although suppuration existed below the skull in both cases, it was not limited in either instance, and therefore would have disappointed the hopes of the surgeon in the second case, had he operated, as it did in the first.

III. Having discussed at such length the relations of suppuration above the dura mater, I do not think I need state equally minutely the appearances in the remaining nine cases, where the pus was situated below that membrane, amongst the mem. branes of the brain. Suffice it to say, that they tell just the same story. They occurred after the most varied injuries: viz., 1 , scalp wound to the bone; 2 , scalp wound with linear fracture; $3,4,5$, fractured base of the skull ; 6 , compound fracture, with laceration of the brain and hernia cerebri; 7,8 , compound fracture with depression, for which the trephine was applied; and 9, a case in which the skull was trephined twelve months after the injury, for the relief of symptoms dependent on depression of the internal table. In none was the suppuration limited. Some were complicated with abscess of the brain; in some the suppuration was doubtless connected with pyæmia, but in a great number of these cases the head only was examined.

IV. Of the fourth class which I have mentioned, viz., that in which the matter was situated only in the cerebrum itself, no instance occurred except the case (Class I, Case III) in which it was unsuccessfully opened by the trephine.

I have thus endeavoured to give my reasons for believing that intracranial suppuration after injuries very rarely occurs in the limited form, and is, in a great number, if not the majority of cases, only one of the manifestations of pyæmia, accompanied or caused by phlebitis of the veins of the diploe. I have no desire, however, to impugn Mr. Dumville's conclusion, that the trephine ought to be used in such cases, wherever no special contraindication exists : only let us remember that the operation is recommended merely as a pis aller, seeing that the disease is, as far as we know, if left to itself necessarily fatal, and that in a few recorded instances the operation has afforded relief.

39, Curzon Street, Mayfair, October 185s.

\section{REMARKS ON MYALGIA: WITH CASES.}

By T. Indran, M.D., Liverpool.

[Continued from page 408.$]$

THE subject of myalgia is at the same time so novel and so interesting, that scarcely any apology is needed for keeping it before the notice of the profession. I have already trespassed frequently on the space of this Journal; but new phases are constantly occurring, giving one a greater idea of the extent and bearing of the subject.

Before recording some fresh cases illustrative of important points, let me recapitulate the chief phenomena of myalgic or muscular pains. They have their seat in the fleshy parts of muscles, in their tendinous prolongations, or in the fibrous aponeuroses. The continuous stretching of any white fibrous tissue, from whatever cause arising, will produce a similar pain. Fibrous tissue is more readily stretched in the weak than in the strong; and the process is attended with greater pain. The pain is described as hot and aching, and is commonly attended with cutaneous soreness, and tenderness on slight pressure. Whenever the pain is due to stretching, it is relieved, though not absolutely cured, by relaxation. The pain is independent entirely of the course of nerves, etc., and is not 